American Journal of Biochemistry and Biotechnology 1 (4): 199-203, 2005

ISSN 1553-3468

(C) 2005 Science Publications

\title{
Use of X-Ray Diffractometry (XRD) for Identification of Fritillaria According to Geographical Origin
}

\author{
${ }^{1}$ Wang Shujun, ${ }^{2} \mathrm{Yu}$ Jinglin and ${ }^{1} \mathrm{Gao}$ Wenyuan \\ ${ }^{1}$ College of pharmaceuticals and Biotechnology, Tianjin University, Tianjin, 300072, China \\ ${ }^{2}$ School of Science, Department of Chemistry, Tianjin University, Tianjin, 300072, China
}

\begin{abstract}
Fritillaria is a traditional Chinese herbal medicine for anti-tussive and expectorant use in China and some other Asian countries. The Fritillaria Chinese medicine material derived from different geographical origins is always difficult to discriminate each other. The objective of the study is to develop a nondestructive and simple method to identify five Fritillaria Chinese materia medica such as Fritillaria thunbergii Miq., Fritillaria ussurensis Maxim., Fritillaria cirrhosa D.Don, Fritillaria pallidifloca Schrenk and Fritillaria hupehensis Hsiao et KC Hsia. X-ray diffraction was utilized to analyze the discrepancy of the Fritillaria from different geographical origins. Because Fritillaria consists of plenty of starch, which is a very good semicrystalline macromolecule, the X-Ray diffraction spectra of Fritillaria powders mainly showed the crystalline properties of starch. The differences in starches crystallinite could be distinguished by X-ray diffraction spectra. X-ray diffraction is proved to be a new and powerful method to discriminate Fritillaria from different geographical origins.
\end{abstract}

Key words: Fritillaria, starch, X-ray, crystal type, degree of crystallinity

\section{INTRODUCTION}

Fritillaria (Chinese name Beimu), the bulbs of various species of the genus Fritillaria (Liliaceae), is a very useful traditional Chinese medicine (TCM) with antitussive and expectorant functions ${ }^{[1-4]}$. There are many kinds of Fritillaria Chinese medicine materials. In China pharmacopoeia (2000 edition), $F$. thunbergii Miq, F. ussurensis Maxim, F. palldifloca Schrenk, $F$. cirrhosa D.Don and F. hupehensis Hsiao et K.C. Hsia are recorded ${ }^{[5]}$. China has a wide range of Fritillaria resources which are distributed extensively. The Fritillatia Chinese medicinal materials from different geographical origin are very easy to confuse, in addition, the size of Fritillaria bulb is much relevant to the growing conditions ${ }^{[6]}$. All of these result in the difficulty in original identification of Fritillaria.

Up to now, chromatographic method is mainly utilized for the identification of different Fritillaria Chinese medicinal materials. Since there are tens of major bioactive components, which are slightly different due to different growing conditions and geographical origins, we can not select only a limited number of specific constituents as essential evaluative criteria. Indeed, there are some contradicting results concerning the contents of some ingredients contained in the Fritillaria in the literature ${ }^{[7,8]}$. In the holistic theory of traditional Chinese medicine, the medicinal materials take effects in curing diseases as a whole. Any method or technique which destroys the wholeness of the traditional Chinese medicine will not be primarily accepted. Recently, there are some methods which keep the integrity of traditional Chinese medicine for discrimination of Fritillaria such as Fourier transform infrared spectroscopy (FTIR), differential scanning calorimetry (DSC) and themogravimetric analysis (TGA) $)^{[9-10]}$. FTIR is a nondestructive, fast and integrity-emphasized method. However, FTIR has the poor character of the fingerprint spectrum. If the Fritillaria are identified clearly, the two-dimensional correlation infrared spectroscopy under thermal perturbation is always utilized. The DSC and TGA methods are carried out under the heating condition and the chemical constituents in the Fritillaria are always destroyed.

X-Ray diffraction has the advantages of strong fingerprint character and nondestruction. The main component in the bulbs of Fritillaria species is starch occupying approximately $80 \%$ content in the total biomass $^{[11]}$. Starch is an important polysaccharide reserve in higher plants. It consists of two main components, amylose and amylopectin. Amylose is an $\alpha$-(1 $\rightarrow 4)$-D-glucopyranosyl polymer, with linear or lightly branched structures or a mixture of both. The residues in amylopectin are $\alpha$-( $1 \rightarrow 4)$-D-glucopyranosse units with $\alpha-(1 \rightarrow 6)$-linkages at intervals of approximately 20 units, depending on plant sources ${ }^{[12-15]}$. Starch is a semicrystalline polymer in which amylose forms the crystalline region and amylopectin forms the amorphous region. As for the Fritillaria powder, the

Corresponding Author: Wang Shujun, College of Pharmaceuticals and Biotechnology, Tianjin University, Tianjin, China, 300072 Tel: $86-22-87401895$ 
starch crystalline diffraction peaks predominate in the $\mathrm{X}$-ray diffraction spectrum.

In this study, X-Ray diffraction method was utilized to identify the Fritillaria from different geographical origins. By analyzing degree of crystallinity of Fritillaria powders and starches as well as their crystal type, we could easily discriminate the Fritillaria Chinese medicinal materials.

\section{MATERIALS AND METHODS}

Sources and Pretreatment of the five Fritillaria samples: Fritillaria thunbergii Miq., Fritillaria ussurensis Maxim., Fritillaria pallidifloca Schrenk, Fritillaria cirrhosa D.Don and F. hupehensis Hsiao et K.C. Hsiawere provided by Meiwei TCM company (Anguo, Hebei province, China) and were identified by Professor Gao Wenyuan, Tianjin University, China.

The five Fritillaria were cleaned, comminuted to powders which were sieved with $160 \mathrm{M}$ sifter and then kept in a desiccator. The dried powders were extracted with $85 \%$ alcohol in a thermostat at $25^{\circ} \mathrm{C}$ for $48 \mathrm{~h}$. The solution was filtrated with an anti-acid filter. The residue was washed with $85 \%$ alcohol for several times and then desiccated at ambient temperature.

X-ray powder diffraction measurements: Monochromatic $\mathrm{Cu}-\mathrm{K}_{\alpha} \square$ radiation (wavelength $=1.542$ $\AA$ ) was produced by a BDX3300 X-ray powder diffractometer (Beijing University Equipment Manufacturer, China). The Fritillaria and starch powders were packed tightly in a rectangular aluminum cell. The samples were exposed to the X-ray beam from $\mathrm{X}$-ray generator running at $36 \mathrm{KV}$ and $20 \mathrm{~mA}$. The scanning regions of the diffraction angle $2 \theta$ were 10 $30^{\circ}$, which covered most of the significant diffraction peaks of the starch crystallites. Other operation conditions included: Step interval 0.02 , scan rate $2 / \mathrm{min}$, Sollet and divergence slit, $1^{\circ}$, receiving slit, $1^{\circ}$ and scattering slit, $0.15^{\circ}$. The same measurements were made at room temperature for three times. Radiation was detected with a proportional detector.

Determination of the degree of crystallinity: The degree of crystallinity of samples was quantitatively estimated following the method of Nara and Komiya ${ }^{[16]}$. A smooth curve which connected peak baselines was computer-plotted on the diffractograms (Fig. 1). The area above the smooth curve was taken as the crystalline portion and the lower area between smooth curve and the linear baseline which connected the two points of the intensity $2 \theta$ of $30^{\circ}$ and $10^{\circ}$ in the samples was taken as the amorphous section. The upper diffraction peak area and the total diffraction area over the diffraction angle $10-30^{\circ}, 2 \theta$ were integrated using Smadchrom software (Morgan and Kennedy Research, Australia). The ratio of upper area to total diffraction was taken as the degree of crystallinity.

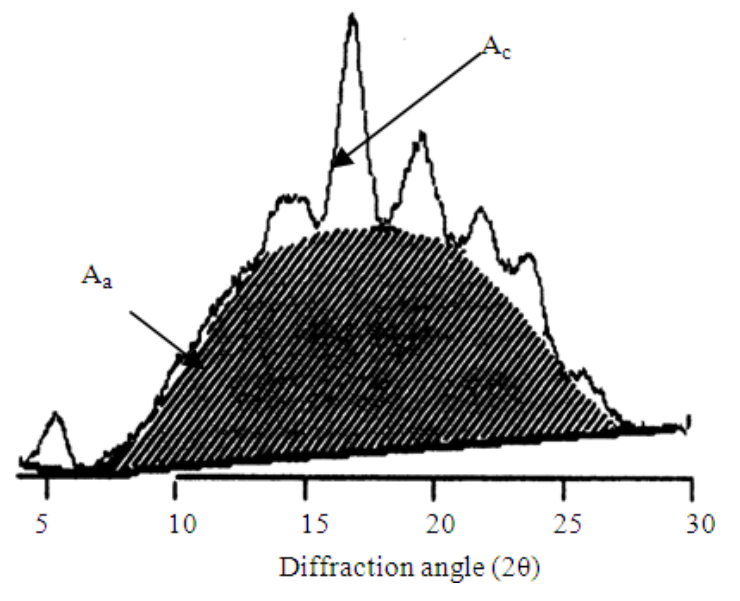

Fig. 1: Calculation of the relative degree of the crystallinity

The equation of the degree of crystallinity is as follows:

$$
X_{c}=A_{c} /\left(A_{c}+A_{a}\right)
$$

Where:

$\mathrm{X}_{\mathrm{c}}=$ refers to the degree of crystallinity

$\mathrm{A}_{\mathrm{c}}=$ refers to the crystallized area on the X-ray diffractogram

$\mathrm{A}_{\mathrm{a}}=$ refers to the amorphous area on the X-ray diffractogram

\section{RESULTS AND DISCUSSION}

X-Ray diffraction analysis of Fritillaria powders: Much of the information about starch granule crystalline properties has been acquired from X-ray powder diffraction studies. Starch can be classified to $\mathrm{A}, \mathrm{B}$ and $\mathrm{C}$ forms ${ }^{[17-19]}$. In the native granular forms, the A form starch is associated mainly with cereal starches, such as maize starch and wheat starch. The Xray patterns of these starches give the stronger diffraction peaks at around $15,17,18$ and $23^{\circ}$. The $\mathrm{B}$ form starch is usually obtained from tuber starches, such as potato starch and canna starch. The strongest diffraction peak of the X-ray diffraction pattern appeared at $17^{\circ} 2 \theta$. And there were also a few small peaks at around $2 \theta$ values of 20,22 and $24^{\circ}$. The $\mathrm{C}$ pattern starch is a mixture of both A and B types, such as smooth-seeded pea starch and various bean starches $^{[20]}$.

Because much starch is contained in the Friitllaria, the X-Ray diffraction pattern of Fritillaria powders mainly showed the crystalline properties of starch. The main crystalline peaks in the X-ray diffraction pattern are attributed to the crystalline peaks of starch.

The X-ray diffractograms of the five Fritillaria powders are presented in Fig. 2. 


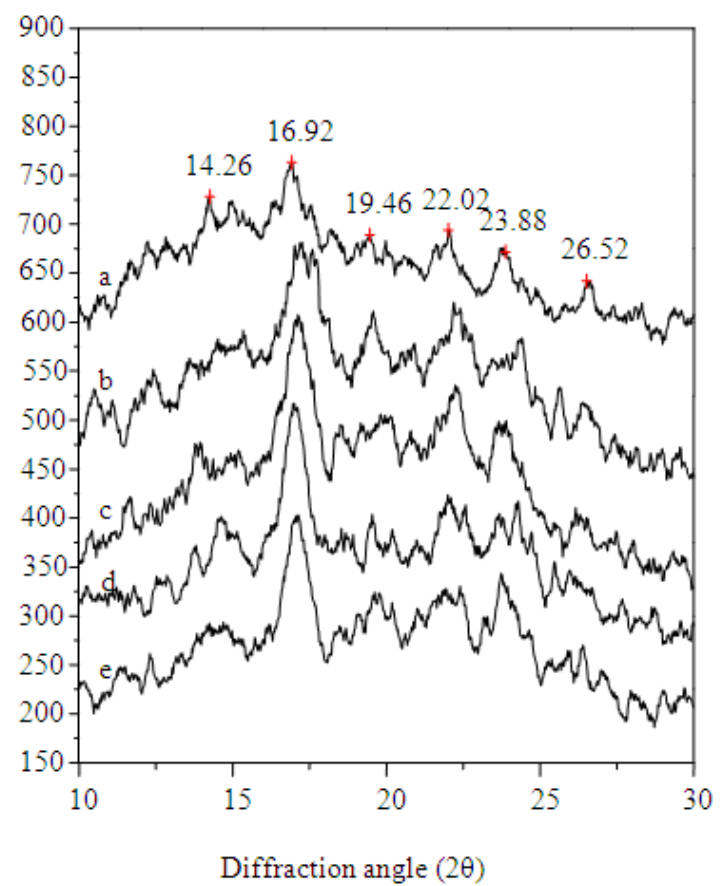

Fig. 2: X-ray diffraction pattern of the five Fritillaria powders, a: F. hupehensis, b: F. pallidifloca, c: $F$. cirrhosa, d: F. ussurensis, e: F. thunbergii

As can be seen from Fig. 2, F. thunbergii, $F$. ussurensis and $F$. cirrhosa powders give the strongest diffraction peak at $17.2^{\circ} 2 \theta$ and a few small peaks at around $2 \theta$ values of $15.3,19.6,22.2,24.4$ and $26.4^{\circ}$. This result revealed that crystal type of starches contained in the three Fritillaria powders is a characteristic B-type. However, the sharp diffraction peak at $17.2^{\circ} 2 \theta$ was converted into two small peaks at 17.2and $17.8^{\circ} 2 \theta$ in the $X$-ray diffraction pattern of pallidifloca powder. This was indicative of A-type, while the other diffraction peaks at 19.6, 22.2, 24.4 and $26.4^{\circ}$ are still characteristic of B pattern. In the light of starch granule crystalline properties, we can conclude that the starch contained in the $F$. pallidifloca was classed as C-type, a mixture of both A and B types. As for the $F$. hupehensis powder, there was a strongest diffraction peak at $16.9^{\circ} 2 \theta$ and two symmetrical peaks at 16.3 and $17.5^{\circ} 2 \theta$ based on the peak top. This phenomenon was firstly found in the study of crystalline properties of starch. And now, we temporarily classed this crystal type of starch as ' $W$ ' form which was not different from the known types.

In term of the above analysis, the five Fritillaria can be sorted into three classes: $F$. thunbergii $F$. ussurensis and $F$. cirrhosa belong to one class, $F$. pallidifloca belongs to another class, $F$. hupehensis belongs to the third class.

There were also apparent differences in the X-ray diffraction pattern of $10-15$ and $25-30^{\circ}$ regions, which were enlarged, respectively, in Fig. 3 and 4.

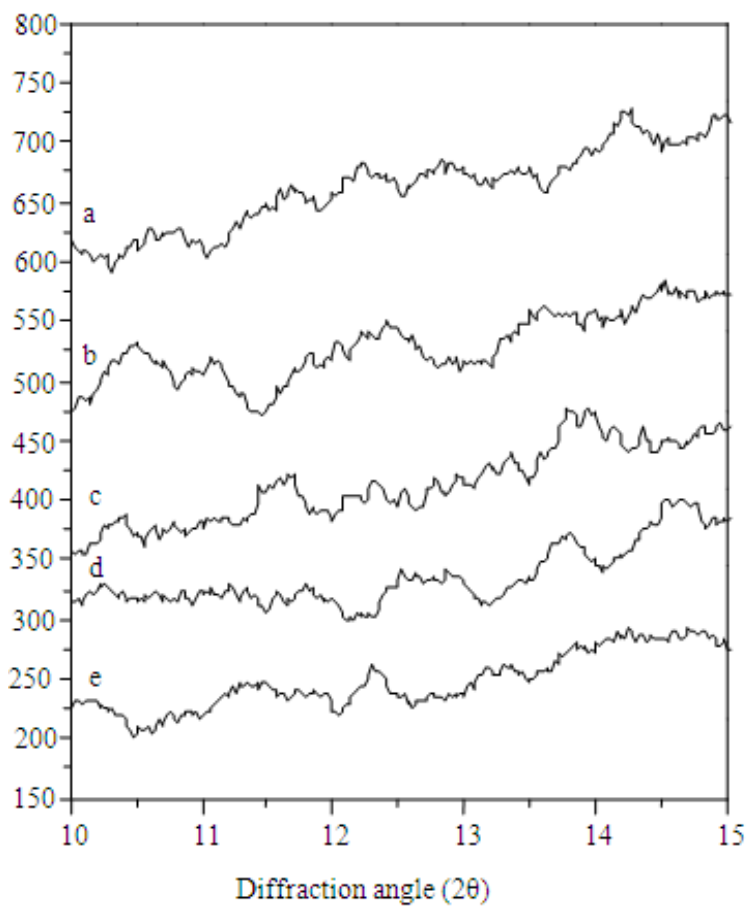

Fig 3: Comparison of the X-ray diffraction pattern of five Fritillaria of 10-15

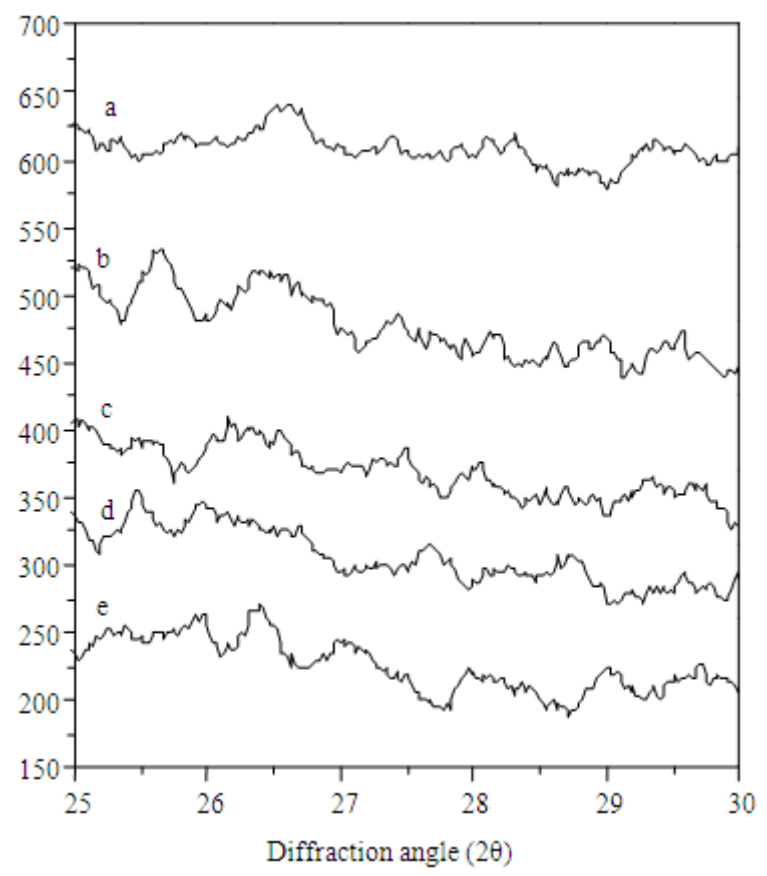

Fig. 4: Comparison of the X-ray diffraction pattern of five Fritillaria of 25-30

The degree of crystallinity of five kinds of Fritillaria powders calculated from the Fig. 2 were shown in Table 1. For this evaluation, we utilized the powders which had almost identical moisture contents $(\sim 12 \%)$ in order to minimize the effect of different moisture contents on crystallinity. 
Table1: X-ray diffraction data of the five Fritillaria powders

\begin{tabular}{lll}
\hline Samples & Degree of crystallinity (\%) & Crystal pattern of starch \\
\hline F. thunbergii & 42.1 & $\mathrm{~B}$ \\
F. ussurensis & 43.6 & $\mathrm{~B}$ \\
F. cirrhosa & 35.9 & $\mathrm{~B}$ \\
F. pallidifloca & 37.7 & $\mathrm{C}$ \\
F. hupehensis & 30.8 & $\mathrm{~W}$ \\
\hline
\end{tabular}

Table 2: X-ray diffraction data of Fritillaria powders after extraction with $85 \%$ alcohol

\begin{tabular}{lll}
\hline Samples & Degree of crystallinity $(\%)$ & Crystal pattern \\
\hline F. thunbergii & 43.2 & $\mathrm{~B}$ \\
F. ussurensis & 40.5 & $\mathrm{~B}$ \\
F. cirrhosa & 41.4 & $\mathrm{~B}$ \\
F. pallidifloca & 44.8 & $\mathrm{C}$ \\
F. hupehensis & 41.4 & $\mathrm{~W}$ \\
\hline
\end{tabular}

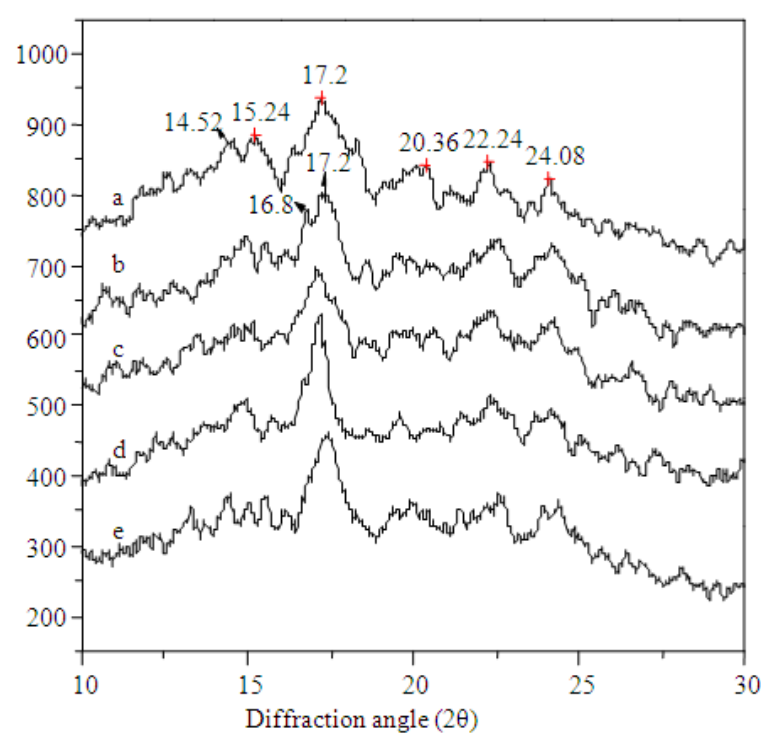

Fig. 5: X-ray diffraction pattern of the five Fritilaria powders after extraction with $85 \%$ alcohol, a: $F$. hupehensis, b: $F$. pallidifloca, c: $F$. cirrhosa, d: F. ussurensis, e: F. thunbergii

According to the degree of crystallinity in Table 1, the five Fritillaria can also be divided into three classes: thunbergii $F$. and ussurensis $F$. belong to one class, cirrhosa $F$. and pallidifloca $F$. belong to another class, hupehensis $F$. belongs to the third class. If the crystal type of starch contained in Fritillaria is considered, the five Fritillaria could clearly be separated into four classes: $F$. thunbergii and $F$. ussurensis belong to one class, $F$. cirrhosa belongs to another class, $F$. pallidifloca belongs to the third class and $F$. hupehensis belongs to the fourth class. In addition, the differences of Fig. 3 and 4 could also provide some information to discriminate $F$. thunbergii and $F$. ussurensis.
$X$-Ray diffraction analysis of Fritillaria powders after extraction with $\mathbf{8 5 \%}$ alcohol: In Fig. 5 X-ray diffraction pattern of the five Fritillaria powders after extraction with $85 \%$ alcohol are displayed.

In order to further test the crystal type of starch contained in Fritillaria, the Fritillaria powder after extraction with $85 \%$ alcohol was analyzed by X-ray diffraction. Consistent with the above analysis, the Xray diffraction pattern of the $F$. thunbergii, $F$. ussurensis and $F$. cirrhosa powders after extraction with $85 \%$ alcohol are the characteristic B-type pattern. The peak at $17^{\circ} 2 \theta$ were stronger than the other peaks at $15,19,22$ and $24^{\circ} 2 \theta$. For $F$. pallidifloca powders after extraction with $85 \%$ alcohol, there were also two small peaks at $16.8^{\circ}$ and $17.2^{\circ} 2 \theta$, which is also an indicative of A-type. The peaks at 15, 19, 22 and $24^{\circ} 2 \theta$ is the characteristic peaks of B-type starch. So, the starch contained in $F$. pallidifloca was classified as Ctype. For $F$. hupehensis powder after extraction with $85 \%$ alcohol, the strongest peak was also at about $17^{\circ}$ $2 \theta$. The two symmetrical peaks centered at $17,16.3$ and $18.2^{\circ} 2 \theta$, respectively. This result further confirmed that the starch contained in the hupehensis $F$. was ' $\mathrm{W}$ ' type that was defined for the moment.

The degree of crystallinity of five kinds of Fritillaria powders after extraction with $85 \%$ alcohol calculated from the Fig. 5 were shown in Table 2. For this evaluation, we utilized the powders which had almost identical moisture contents $(\sim 15 \%)$ in order to minimize the effect of different moisture contents on crystallinity.

\section{CONCLUSION}

The study showed the differences in starch contained in different Fritillaria of geographical origins. According to these discrepancies in starch crystal type and crystallinity of Fritillaria powder, we could easily separate the five Fritillaria. As a new analytical technology, X-ray diffraction method was widely utilized in the study of phase and crystal structure of substance. Diffraction pattern obtained by this method could provide lots of information, strong fingerprint character, stability and reliability. X-ray diffraction could be utilized as identification method of all kinds of traditional Chinese medicine. This experiment not only gave some crystallinite properties on starch contained in Fritillaria, but provided some information on the plant taxonomy from macromolecule aspects.

\section{REFERENCES}

1. Li S.L., S.W. Chan, P. Li, G. Lin and G.H. Zhou et al., 1999. Pre-column derivatization and gas chromatographic determination of alkaloids in bulbs of Fritillaria. J. Chromatogr. A., 859: 183-192. 
2. Li S.L., P. Li, G. Lin, S.W. Chan and Y.P. Ho, 2000. Simultaneous determination of seven major isosteroidal alkaloids in bulb of Fritillaria by gas chromatography. J. Chromatogr. A., 873: 221-228.

3. Lin G., P. Li, S.L. Li and S.W. Chan, 2001. Chromatographic analysis of Fritillaria isosteroidal alkaloids, the active ingredients of Beimu, the antitussive traditional Chinese medicinal herb. J. Chromatogr. A., 935: 321-338.

4. Li S.L., G. Lin, S.W. Chan and P. Li, 2001. Determination of the major isosteroidal in bulbs of Fritillaria by high-performance liquid chromatography coupled with evaporative light scattering detection. J. Chromatogr. A., 909: 207-214.

5. Zheng, X.Y. and X. Li, 2000. Pharmacopoeia of the People's Republic of China. Part II. Chemistry and Chemical Engineering Press, Beijing.

6. Gao, W. and Z. Li, 1994. The anatomical study of Fritillaria Thunbergii in the senescent process, Guangxi Plant, 14: 65-69.

7. Xiao, P., 2002. Modern Chinese Materia Medica. Chemistry Industry Press, pp: 114.

8. Song, F., Q. Zhao and W. Xing, 1997. Comparison of total alkaloids content of Fritillaria from different origin. J. Huaxi Med., 12: 259-261.

9. Hua, R., S. Sun and Q. Zhou et al., 2003. Discrimination of Fritillary according to geographical origin with Fourier transform infrared spectroscopy and two-dimensional correlation IR spectroscopy. J. Pharmaceut. Biomed. Anal., 33: 199-209.

10. Lu Y., Q.T., Zheng, N. Wu et al., 1997. Studies on $\mathrm{X}$-ray diffraction pattern of traditional Chinese medicinal material. Acta Pharmaceut. Sinica, 92: 193-198.

11. Gao, W., L. Fan and KY. Paek, 1999. Ultrastructure of amyloplasts and intrecellular transport of old and new scales in Fritillaria ussuriensis. J. Plant Biol., 42: 117-123.

12. Lelou, V.M., P. Colonna and S.G. Ring, 1991. $\alpha-$ amylase adsorption on starch crystallites. Biotechnol. Bioeng., 38: 127-134.

13. Jacobs, H., N. Mischenko, M.H.J. Koch, C.E. Relinde, D. Jan and R. Harry, 1998. Evaluation of the impact of annealing on gelatinization at intermediate water content of wheat and potato starches: A differential scanning calorimetry and small angle X-ray scattering study. Carbohydrate Res., 306: 1-10.
14. Paris, M., H. Bizot, J. Emery, J.Y. Buzaré and A. Buléon, 1999. Crystallinity and structuring role of water in native and recrystallized starches by $13 \mathrm{C} \mathrm{CP-MAS}$ NMR spectroscopy: 1: Spectral decomposition. Carbohydrate Polymers, 39: 327-339.

15. Waigh, T.A., A.M. Donald, F. Heidelbach, C. Riekel and M.J. Gidley, 1999. Analysis of the native structure of starch granules with small angle x-ray microfocus scattering. Biopolymers, 49: 91-105.

16. Nara, S. and T. Komiya, 1983. Studied on the relationship between water-saturated state and crystallinity by the diffraction method for moistened potato starch. Starch-Starke, 35: 407-410.

17. Zobel, H., 1988. Starch crystal transformations and their industrial importance. Starch-Starke, 40: 1-7.

18. Cheetham, N.W.H. and L. Tao, 1998. Variation in crystalline type with amylose content in maize starch granules: an X-ray powder diffraction study. Carbohydrate Polymer, 36: 277-284.

19. Hizukuri, S., 1985. Relationship between the distribution of the chain length of amylopectin and the crystalline structure of starch granules. Carbohydr Res., 141: 295-306.

20. Elsenhaber, F. and W. Schulz, 1988. Monte carlo simulation of the hydration shell of double-helical amylose: A left-handed antiparallel double helix fits best into liquid water structure. Biopolymers, 27: 1205-1221. 